\title{
Larger Genomes Linked to Slower Development and Loss of Late-Developing Traits
}

\author{
Molly C. Womack, ${ }^{1, \star}$ Marissa J. Metz, ${ }^{1,2}$ and Kim L. Hoke ${ }^{1}$ \\ 1. Department of Biology, Colorado State University, Fort Collins, Colorado 80523; 2. Department of Biomedical Sciences, Colorado State \\ University, Fort Collins, Colorado 80521 \\ Submitted September 25, 2018; Accepted June 3, 2019; Electronically published October 18, 2019 \\ Online enhancements: appendix. Dryad data: https://doi.org/10.5061/dryad.k02pq01.
}

\begin{abstract}
AвSTRACт: Genome size varies widely among organisms and is known to affect vertebrate development, morphology, and physiology. In amphibians, genome size is hypothesized to contribute to loss of lateforming structures, although this hypothesis has mainly been discussed in salamanders. Here we estimated genome size for 22 anuran species and combined this novel data set with existing genome size data for an additional 234 anuran species to determine whether larger genome size is associated with loss of a late-forming anuran sensory structure, the tympanic middle ear. We established that genome size is negatively correlated with development rate across 90 anuran species and found that genome size evolution is correlated with evolutionary loss of the middle ear bone (columella) among 241 species (224 eared and 17 earless). We further tested whether the development of the tympanic middle ear could be constrained by large cell sizes and small body sizes during key stages of tympanic middle ear development (metamorphosis). Together, our evidence suggests that larger genomes, slower development rate, and smaller body sizes at metamorphosis may contribute to the loss of the anuran tympanic middle ear. We conclude that increases in anuran genome size, although less drastic than those in salamanders, may affect development of late-forming traits.
\end{abstract}

Keywords: trait loss, evo-devo, heterochrony, paedomorphosis, tympanic middle ear loss, earless.

\section{Introduction}

Genome size evolution alters development, morphology, and physiology across vertebrates. Specific consequences of larger genomes include larger cell size (Ohno 1970; Szarski 1970, 1983; Olmo and Morescalchi 1975, 1978; Horner and Macgregor 1983; Walker et al. 1991; Gregory 2000, 2001, 2002a; Hardie and Herbert 2003; Mueller et al. 2008; Roth and Walkowiak 2015), slower metabolic rate (Vinogradov 1995, 1997; Gregory

\footnotetext{
* Corresponding author. Present address: Department of Vertebrate Zoology, National Museum of Natural History, Smithsonian Institution, Washington, DC 20560; email: mollywo@berkeley.edu.

ORCIDs: Womack, https://orcid.org/0000-0002-3346-021X; Hoke, https:// orcid.org/0000-0001-5553-0138.

Am. Nat. 2019. Vol. 194, pp. 854-864. (C) 2019 by The University of Chicago. 0003-0147/2019/19406-58760\$15.00. All rights reserved. DOI: $10.1086 / 705897$
}

2002a), and slower development rate (Goin et al. 1968; Bachmann 1972; Bennett 1977; Oeldorf et al. 1978; Horner and Macgregor 1983; Camper et al. 1993; White and McLaren 2000; Chipman et al. 2001; Roth and Walkowiak 2015; Liedtke et al. 2018). Within amphibians, larger genome size and the associated larger cell sizes (Horner and Macgregor 1983; Roth et al. 1994; Gregory 2001; Mueller et al. 2008; Roth and Walkowiak 2015) have been hypothesized to affect the development of late-forming anatomical structures via two potential mechanisms. Larger cells take longer to proliferate (Shuter et al. 1983; Vinogradov 1999), which is thought to contribute to slower embryonic development (Bachmann 1972; Oeldorf et al. 1978; Horner and Macgregor 1983; Chipman et al. 2001), larval development (Goin et al. 1968; Camper et al. 1993), and limb regeneration (Sessions and Larson 1987) in amphibians with larger genomes. This slowing of development rate (neoteny) can lead to paedomorphosis, a form of heterochrony (change in developmental timing) in which organisms become sexually mature before all traits have reached their adult phenotype (Wake 1966; Gould 1977; Alberch et al. 1979; McNamara 1986). However, it also possible that larger cell sizes associated with large genomes constrain development at small body sizes due to limits on cell number, as proposed in salamanders (Wake 1991; Hanken and Wake 1993). However, the relationship between larger genome size and evolutionary lability of late-forming traits has limited empirical support.

Most work focused on the association between genome size and trait loss has occurred in salamanders, which have an extreme range of genome sizes (from 10.1 pg in Gyrinophilus porphyriticus to 120.6 pg in Necturus lewisi; Gregory 2018) that are sometimes accompanied by toe and lung loss (Wake 2009). However, frogs and toads (anurans) provide an excellent opportunity to test whether less extreme genome size evolution could affect the development of late-forming structures. Genome size varies in anurans (from $0.95 \mathrm{pg}$ in Limnodynastes ornatus to $19 \mathrm{pg}$ in Arenophryne rotunda; Gregory $2002 b$ ) and is positively associated with length of embryonic (Bachmann 1972; Oeldorf et al. 1978; Horner and Macgregor 
1983; Chipman et al. 2001) and larval (Goin et al. 1968; Camper et al. 1993) development in the limited taxa studied to date. Furthermore, Roth et al. (1994) and Roth and Walkowiak (2015) found that anurans (and salamanders) with larger genome size had lower complexity of late-forming brain structures. Thus, genome size variation in anurans may alter morphology, although the prevalence of such morphological changes and the underlying developmental mechanisms remain unclear.

One late-forming structure that may be affected by genome size evolution in anurans is the tympanic middle ear. The tympanic middle ear facilitates detection of airborne sounds and is a key tetrapod adaptation for hearing on land (reviewed in Manley 2010). Surprisingly, within anurans, the tympanic middle ear has been lost at least 38 times (Pereyra et al. 2016) despite reduction of high-frequency (above $1 \mathrm{kHz}$ ) hearing sensitivity (Lombard and Straughan 1974; Womack et al. 2017) and lack of clear environmental selection pressures to explain tympanic middle ear loss (Jaslow et al. 1988). Furthermore, some frogs that are earless (completely lack all tympanic middle ear structures) or have reduced tympanic middle ears, such as Bombina bombina and B. orientalis, also have large genomes (Roth and Walkowiak 2015). Therefore, we hypothesize that the loss of peripheral auditory structures in anurans may result from larger genome sizes, which either result in paedomorphic loss of the tympanic middle ear or result in larger cell sizes that impose space constraints on small-bodied anurans.

The tympanic middle ear forms quite late, making it vulnerable to genome size expansion and associated shifts in development rate, such as paedomorphosis. Development of the middle ear structures begins just prior to metamorphosis and proceeds proximally to distally (Hetherington 1987; Vorobyeva and Smirnov 1987; Smirnov 1991; Womack et al. $2018 b$ ). Middle ear structures are lost in a distal to proximal fashion; hence, loss may reflect incomplete development (Vorobyeva and Smirnov 1987; Smirnov 1991; Pereyra et al. 2016). Furthermore, the middle ear can take up to a year to complete development (Sedra and Michael 1959), leaving it vulnerable to processes that truncate development of lateforming structures.

Ontogenetic data from a few species implicate various forms of paedomorphosis (neoteny, progenesis, and postdisplacement) as an explanation for tympanic middle ear loss. For neoteny, development rate of the organism is slowed and late-forming traits are truncated. If progenesis occurs, the development rate does not change but development of the animal ends early and late-forming traits are truncated. Postdisplacement entails the delayed onset of a specific trait's development and truncation of the trait's development. Van Eeden (1951) first hypothesized the truncated middle ear development in the coastal tailed frog (Ascaphus truei) was a case of neoteny. Vorobyeva and Smirnov (1987) compared middle ear development at sparse ontogenetic time points among nine anuran species and attributed middle ear variation to truncated development (progenesis). Smirnov (1991) compared tympanic middle ear development in 10 anuran species and argued that three different paedomorphic mechanisms could explain middle ear variation in three families (Microhylidae, progenesis; Bombina, neoteny; Bufonidae, postdisplacement) based on lineage-specific differences in developmental timing of cranial structures. However, none of these studies explicitly tested for differences in development rate among species to determine whether slower development rate (neoteny) could explain middle ear loss. One reason development rate has not been rigorously compared between species that vary in middle ear presence/absence is a lack of development rate data for earless species. However, genome size, which is more easily measured and correlates positively with length of embryonic (Horner and Macgregor 1983) and larval (Goin et al. 1968) development in the few anurans species studied, may provide indirect evidence that slower development promotes middle ear loss.

A non-mutually-exclusive mechanism by which genome size could affect lability of late-forming structures is cell size to body size ratios that constrain the space available for structures to form. Genome sizes are associated with larger cell sizes in anurans (Olmo and Morescalchi 1978; Horner and Macgregor 1983; Gregory 2001; Mueller et al. 2008; Roth and Walkowiak 2015) as well as all other vertebrates (Ohno 1970; Szarski 1970, 1983; Olmo and Morescalchi 1975, 1978; Walker et al. 1991; Gregory 2000, 2001, 2002a; Hardie and Herbert 2003). Wake (1991) and Hanken and Wake (1993) proposed that large cell sizes and small body sizes prevent formation of late-developing peripheral digits in salamanders. Furthermore, the large cells and small bodies of the salamander Thorius appear to generate a developmental tradeoff between the eyes and the brain, resulting in deformation and posterior placement of the brain (Hanken 1983; Wake 1991). The anuran middle ear emerges just before metamorphosis (Sedra and Michael 1959; Hetherington 1987; Vorobyeva and Smirnov 1987; Horowitz et al. 2001; Womack et al. 2018b), and size at metamorphosis varies widely among species (from $5 \mathrm{~mm}$ in Dendrophryniscus minutus to $95 \mathrm{~mm}$ in Rana catesbeiana; Collins 1979; Moreira and Lima 1991). Thus, large cell sizes in combination with smaller size at metamorphosis may disrupt development of the late-forming peripheral auditory system of anurans.

Here we assessed the relationship among anuran genome size, length of the larval period, and middle ear loss within a phylogenetic context. We combined genome sizes measured in this study with existing genome size and development rate data to estimate the correlation between genome size and length of the larval period while accounting for phylogenetic relationships. We then used phylogenetic comparative analyses to determine whether larger genome size is associated 
with middle ear loss by comparing genome size between eared species, which develop a full columella (middle ear bone), and earless species, which lack all tympanic middle ear structures. We demonstrated a positive relationship between genome size and cell size among eared and earless anurans and used phylogenetic comparative analyses to determine whether earless species have smaller body sizes at metamorphosis in comparison to eared species. Finally, we discuss the lack of shared life-history or ecological traits among the earless species in our study, demonstrating little evidence for alternative explanations for tympanic middle ear loss. Our results expand on previously observed relationships among genome size, development rate, and cell size in anurans and link genome size evolution with evolutionary lability of the anuran tympanic middle ear.

\section{Material and Methods}

\section{Animal Collection and Museum Specimens Used for Feulgen Staining}

In this study, we used Feulgen staining to image red blood cells and estimate the genome sizes of fixed specimens, both field caught and from museum collections. We captured four anuran species (two earless species, two eared species, seven total animals) from sites in Ecuador and Peru (see table A1 for details; tables A1-A3 are available online). We euthanized live-caught animals with 20\% topical benzocaine and then decapitated the animals. The specimen's bodies were preserved in $70 \%$ ethanol. The Institutional Animal Care and Use Committee at Colorado State University approved all experiments (protocol 12-3484A), and the Ministerio del Ambiente in Ecuador and the Servicio Forestal y de Fauna Silvestre in Peru approved collection, research, and export permits. We added another 18 species (six earless species, 12 eared species, 24 total specimens) by using formalin-fixed specimens from the United States National Museum (see table A2 for details). We classified species as earless if they lacked all tympanic middle ear structures (columella, tympanic annulus, and tympanic membrane) according to Pereyra et al. (2016), and we excluded species that had conflicting reports with regard to presence/absence of the columella (Telmatobius niger) or that had only been reported to have a very small portion of the columella present (Bombina orientalis and Bombina variegata), as these species could not be clearly categorized as eared or earless.

\section{Feulgen Staining for Estimation of Genome and Cell Size}

We stained and quantified genome size using Feulgen staining of chromatin as described elsewhere (Hardie et al. 2002). In brief, we extracted erythrocytes from preserved specimens (both ethanol and formalin fixed, as described above). We then air-dried the slides, fixed them in MFA (methanol to formalin to acetic acid, $85: 10: 5$ ) for $24 \mathrm{~h}$, rinsed them for $10 \mathrm{~min}$ under running tap water, hydrolyzed them in $5.0 \mathrm{~N} \mathrm{HCl}$ for $2 \mathrm{~h}$, briefly rinsed them in $0.1 \mathrm{~N} \mathrm{HCl}$, stained them for $2 \mathrm{~h}$ in Schiff reagent, rinsed them three times for $5 \mathrm{~min}$ each in bisulfite solution, and then rinsed them in tap water. After three 2-min rinses in distilled water, we air-dried the slides and applied coverslips with Permount. We white balanced and imaged slides and then calculated integrated densities of 2 27 erythrocyte nuclei (mean, 13.05; SD, 7.65) as a measure of genome size, and we measured the area of three red blood cells per specimen using Fiji (Schindelin et al. 2012). We then calculated an average integrated density and red blood cell size for each specimen and averaged those values within species to estimate genome size and red blood cell size for all species.

\section{Statistical Analyses}

We performed all data visualization and statistical analyses for this study in R (R Core Team 2017). All data and associated references are available in the Dryad Digital Repository (https://doi.org/10.5061/dryad.k02pq01; Womack et al. 2019). Before testing for differences in genome size between eared and earless species, we first verified the accuracy of the genome sizes we estimated with Feulgen staining by generating a standard curve. The standard curve included species' averages of genome size for eight species within our Feulgen staining study that also had previously published genome sizes in the Animal Genome Size Database (Gregory 2018). We found that our genome size estimates were tightly correlated with previously reported genome sizes $\left(R^{2}=0.925, F_{1,6}=\right.$ 73.97, $P<.001)$, indicating that our genome size estimates were reliable. For downstream analyses, we used this standard curve regression to convert each species' average integrated density recorded from Feulgen staining to a $C$ value that was comparable with averaged species genome sizes from the Animal Genome Size Database as well as a recently published study (Liedtke et al. 2018).

To test for an association between genome size and length of the larval period, we ran a phylogenetic generalized least squares (PGLS) analysis using the R packages nlme (Pinheiro et al. 2017) and phytools (Revell 2012). We first trimmed a recent phylogeny of amphibians (Pyron 2014a, 2014b) to the 90 species for which we had both genome size estimates and records of minimum larval period. We chose minimum larval period because larval period can be delayed due to many environmental factors, including diet (Pfennig et al. 1991; Kupferberg et al. 1994) and temperature (Marian and Pandian 1985; Alvarez and Nicieza 2002; Buchholz and Hayes 2002). We also removed four outlier species (Ascaphus truei, Rana (=Lithobates) grylio, $R$. (=Glandirana) rugose, and $R$. (=Lithobates) septentrionalis) from this analysis because these species only had recorded larval periods that included 
overwintering (300 days or greater). We first tested for phylogenetic signal in the regression residuals for genome size and minimum larval period using the $\mathrm{R}$ package phytools (Revell 2012) to assess whether accounting for phylogenetic relationships in our analysis was appropriate (Revell 2010). We found the residuals of minimum larval period and genome size had significant phylogenetic signal $(K=0.220$, $P=.005)$, so we ran our PGLS analysis with development rate (minimum larval period in days) as the dependent variable and with genome size (average $\mathrm{C}$ value) as the independent variable, and we estimated phylogenetic signal $(\lambda)$ in the residual error simultaneously with the regression parameters (Revell 2010). We ran an additional PGLS analysis with an Ornstein-Uhlenbeck (OU) model, which assumes that the trait is drawn toward a fitness optimum, but we report results only from our better-fit, $\lambda$-transformed model, as determined by Akaike information criterion (AIC) comparison (Brownian motion [BM] AIC 153.03 < OU AIC 163.28; table A3).

We performed another PGLS analysis to assess whether genome size (average $\mathrm{C}$ value) was associated with earlessness. We again trimmed a recent phylogeny of amphibians (Pyron $2014 a, 2014 b$ ) to 241 species with known genome size and middle ear presence/absence. We tested for phylogenetic signal in the regression residuals of genome size and middle ear presence/absence and found significant phylogenetic signal $(K=0.406, P<.001)$. Thus, we ran a PGLS analysis to test for a relationship between genome size (average $\mathrm{C}$ value) and middle ear presence/absence while simultaneously estimating phylogenetic signal $(\lambda)$ in the residual error and the regression parameters (Revell 2010). We ran an additional PGLS analysis assuming an OU model, but we report results only from our better-fit, $\lambda$-transformed model as determined by AIC comparison (BM AIC $894.41<$ OU AIC 1,003.26; table A3).

To determine whether the observed relationship between genome size and tympanic middle ear loss was robust, we performed two resampling tests. Our first resampling test determined whether the differences between eared and earless species seen in this study are robust to changes in earless sampling. We ran 17 additional PGLS analyses testing the relationship between genome size (average $C$ value) and middle ear presence/absence while estimating phylogenetic signal $(\lambda)$ in the residual error simultaneously with the regression parameters (Revell 2010). Each PGLS analysis had one of the 17 earless species removed from the data set. All of the 17 PGLS analyses found an association between larger genome sizes and middle ear absence when accounting for phylogenetic relationships (range of $P$ values, .001-.038). For our second resampling test, we addressed whether the observed relationship between larger genome size and earless species could be influenced by the phylogenetic distribution of genome sizes in eared species, such that smaller genome size estimates for ancestral eared species inflated the observed re- lationship between earlessness and larger genome size. To test this possibility, we randomized genome sizes among only the eared species in our data set to generate 1,000 unique randomized eared species genome size data sets (not modifying genome sizes of earless species from their original values). We then performed 1,000 PGLS analyses with these randomized eared species genome size data sets, simultaneously estimating the regression parameters and phylogenetic signal $(\lambda)$ in the residual error (Revell 2010), and found that $100 \%$ of the 1,000 PGLS analyses reported a significant association between larger genome sizes and middle ear absence when accounting for phylogenetic relationships. This demonstrates that the association between larger genome sizes and tympanic middle ear absence are robust to changes in the phylogenetic distribution of the eared species' genome sizes.

Finally, we estimated the relationship between genome size and cell size and tested whether earless species metamorphosed at smaller body sizes than eared species. We used a linear regression (not PGLS) to estimate the relationship between genome size and red blood cell size because the regression residuals of genome size and cell size showed no phylogenetic signal ( $K=0.402, P=.201)$. Next, we performed PGLS analyses to test for differences between eared and earless species in body size at metamorphosis (minimum and maximum). We ran all downstream tests using minimum recorded body size at metamorphosis and a second identical set of tests that used maximum recorded body size at metamorphosis, and we report the results for both. We trimmed an amphibian phylogeny (Pyron 2014a, 2014b) to the species for which we had records of body size at metamorphosis (both minimum and maximum in millimeters) and middle ear presence/absence. We tested whether the earless species had smaller minimum and maximum body sizes at metamorphosis compared with eared species using PGLS analyses because the regression residuals of the log metamorph body size and middle ear presence/absence showed significant phylogenetic signal (minimum metamorph size: $K=0.466, P<.001$; maximum metamorph size: $K=0.341, P<.001)$. We used the log of metamorph body size in all PGLS analyses, and again we estimated phylogenetic signal $(\lambda)$ in the residual error simultaneously with the regression parameters (Revell 2010). We ran additional PGLS analyses assuming an OU model, but we report results only from our better-fitting, $\lambda$-transformed models as determined by AIC comparison (minimum metamorph size: BM AIC 86.99 < OU AIC 133.66; maximum metamorph size: BM AIC 138.02 < OC AIC 160.09; table A3).

\section{Results \\ Relationship between Genome Size and Length of Larval Period}

Both genome size and minimum larval period varied widely among 90 anuran species included in this study (fig. 1). 


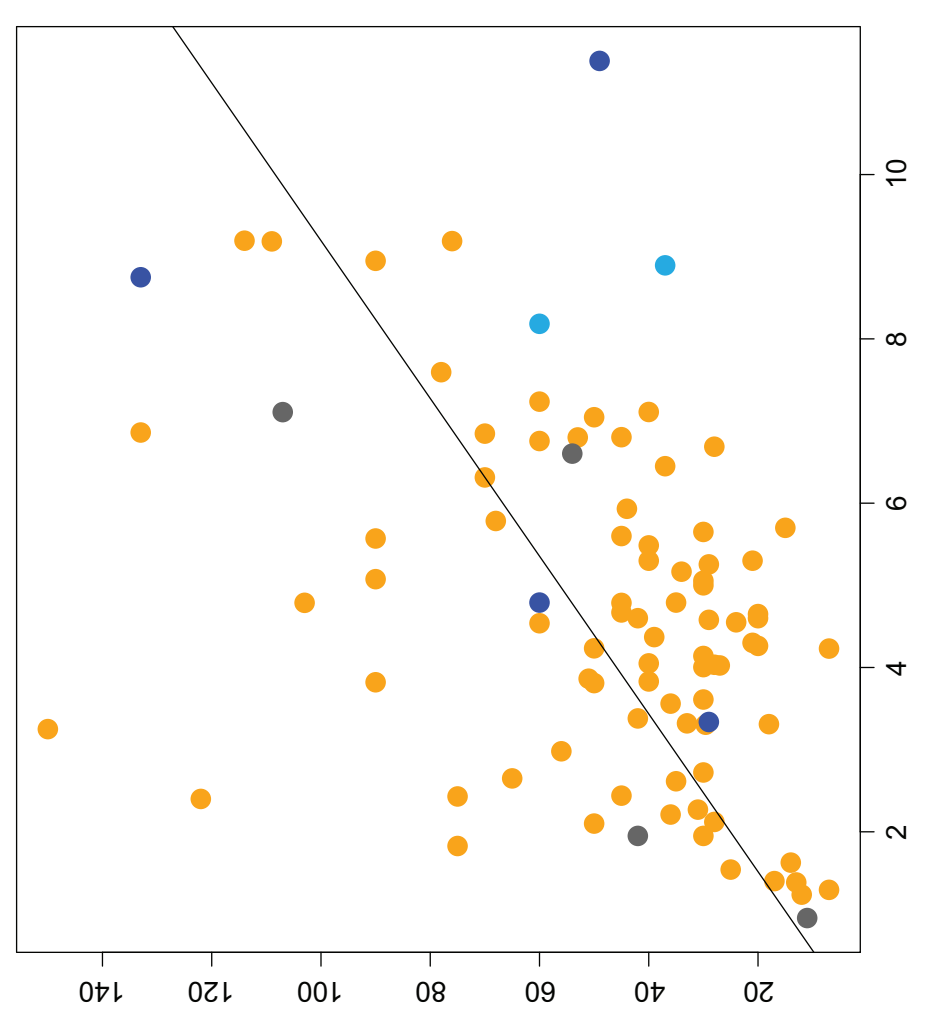

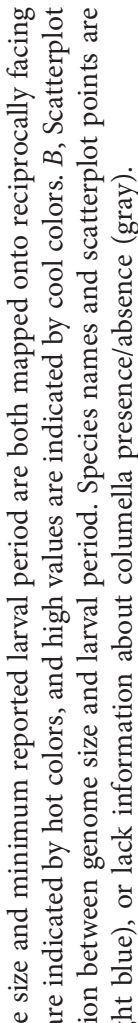

है

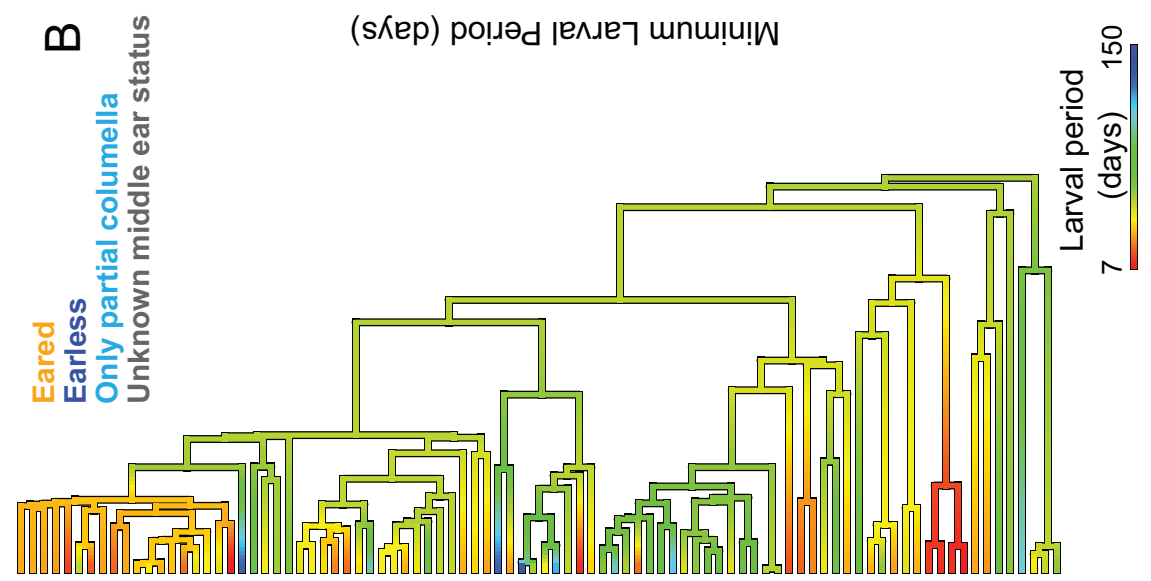

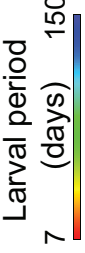

S

स 合号

预

층 ज्ञ

훈

焉焉

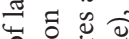

言



可 㞼

$3 . \overline{\mathrm{s}}$

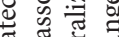

政
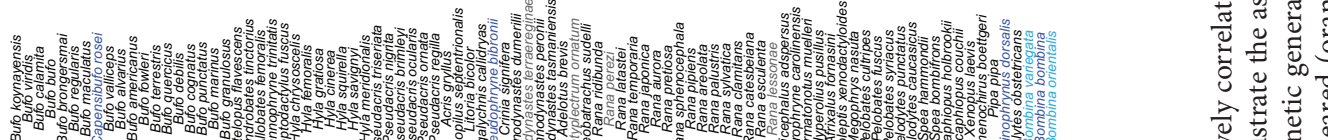

㻤

这

응을 흔

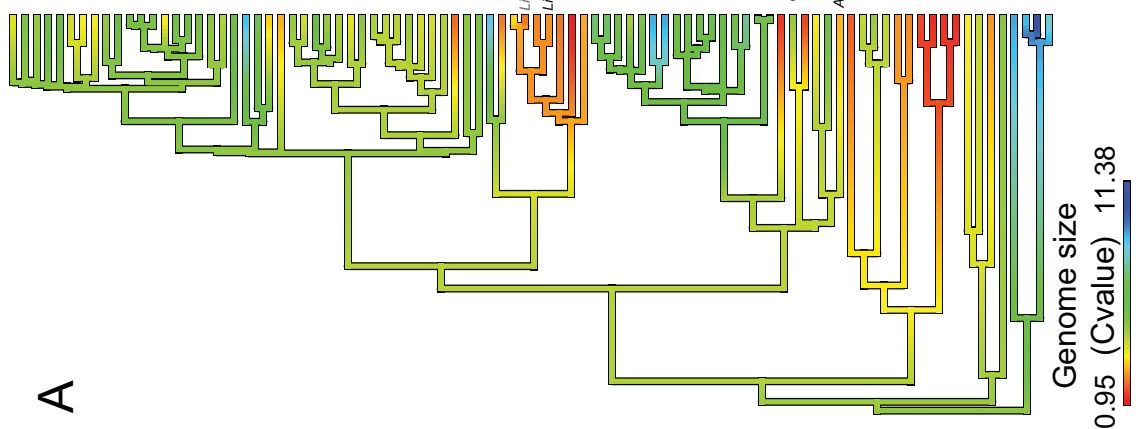

要者

要

品

동ำ

妾家

4.

$\ddot{*}{ }^{\circ}$

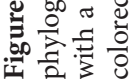


Genome sizes ranged from a C value of 0.95 in Platyplectrum ornatum to 11.38 in Bombina bombina. Minimum reported larval period ranged from 7 days in Scaphiopus couchii and Bufo ( = Rhinella) granulosus to 150 days in Limnodynastes dumerilii. Genome size and minimum larval period had a positive relationship when accounting for phylogenetic relationships $\left(F_{1,88}=19.6, P<.001\right.$; fig. 1 ; table A3). Although species with larger genomes tended to have longer minimal larval periods, a small number of species with small genomes had long larval periods, such as Limnodynastes tasmaniensis, which had a $\mathrm{C}$ value of 2.4 and a minimum recorded larval period of 122 days (fig. 1).

\section{Differences in Genome Size between Eared and Earless Anuran Species}

Genome sizes of the 224 eared species ranged from a $C$ value of 1.09 in Petropedetes cameronensis to 11.36 in Rana tientaiensis, and those of the 17 earless species ranged from a C value of 1.81 in Sooglossus sechellensis to 11.38 in Bombina bombina (fig. 2). Although eared and earless species overlapped in genome size, the genomes of earless species were larger on average than the genomes of eared species when accounting for phylogenetic relationships $\left(F_{1,239}=7.6, P=\right.$ .006; table A3; fig. 2).

Genome Size, Cell Size, and Body Size at Metamorphosis

We found a positive relationship between genome size and average red blood cell size for 30 anuran species $\left(F_{1,28}=\right.$ 24.8, $P<.001$; table A3; fig. 3). Next, we compared both minimum and maximum recorded metamorph body size between six earless (Ascaphus truei, Bombina bombina, Capensibufo rosei, Dendrophryniscus minutus, Pseudophryne bibronii, and Rhinophrynus dorsalis) and 76 eared species. Body size at metamorphosis ranged 3.1 to $95 \mathrm{~mm}$ in eared species and from 5 to $41 \mathrm{~mm}$ in earless species (fig. 4). For minimum recorded body size at metamorphosis, earless species had smaller metamorph body sizes than eared species $\left(F_{1,82}=\right.$ 5.3, $P=.023$; table A3; fig. 4). However, maximum size at metamorphosis for earless species trended smaller but did not differ from eared species maximum size at metamorphosis $\left(F_{1,82}=3.3, P=.072\right.$; table A3).

\section{Discussion}

Our results link genome size evolution with the loss of a lateforming sensory structure in anurans, showing that genome size evolution may have sensory consequences in anurans. We demonstrated a positive relationship between the length of the larval period and genome size within anurans, providing a comprehensive test of the link between genome size and development rate while accounting for phylogenetic relationships. We also provide evidence that larger genome sizes may influence tympanic middle ear loss by two nonmutually-exclusive mechanisms: heterochrony or comparatively larger cell sizes and small body sizes during key stages of tympanic middle ear development.

\section{Does Genome Size Limit Development Rate?}

We found that species with larger genome sizes had slower development rates (longer larval periods), similar to previous studies in fewer anuran taxa (Goin et al. 1968; Camper et al. 1993); however, the relationship between genome size and development rate in amphibians is more complex than a linear relationship. Our data suggest that genome size sets a lower limit on the length of the larger period, such that a species with a larger genome cannot develop as fast as a species with a smaller genome; however, species with small genomes may still develop relatively slowly (Limnodynastes tasmaniensis: $C$ value of 2.4, minimum larval period of 122 days; RichterBoix et al. 2011; Gregory 2018). The result that genome size does not accurately predict development rate but instead limits how fast development can proceed was also found by Oeldorf et al. (1978) when they tested the relationship between genome size and duration of embryonic anuran development. Thus, the negative relationship between genome size and development rate remains evident at both embryonic and larval development stages and remains strong with the increased sample size and anuran diversity tested here. Environmental factors, including prey availability (Pfennig et al. 1991; Kupferberg et al. 1994) and temperature (Marian and Pandian 1985; Alvarez and Nicieza 2002; Buchholz and Hayes 2002), could also contribute to the nonlinear relationship between genome size and development rate that we observed. To accomplish a broad phylogenetic comparison, we wanted to compare the fastest larval development that been observed among species. But experimental and comparative studies that control for other factors known to affect development rate (temperature, diet, density, etc.) may better determine how much genome size contributes to the variation length of larval development among anuran species.

\section{Support for Heterochrony Affecting Tympanic Middle Ear Loss}

The differences in genome size between closely related eared and earless species give indirect evidence that heterochrony-specifically neoteny-could be involved in convergent loss of anuran tympanic middle ears. Since genome size shows a positive relationship with minimum larval period, species with larger genomes are less likely to develop as fast as species with smaller genomes. The slowing of development rate (neoteny) in earless species could lead to 


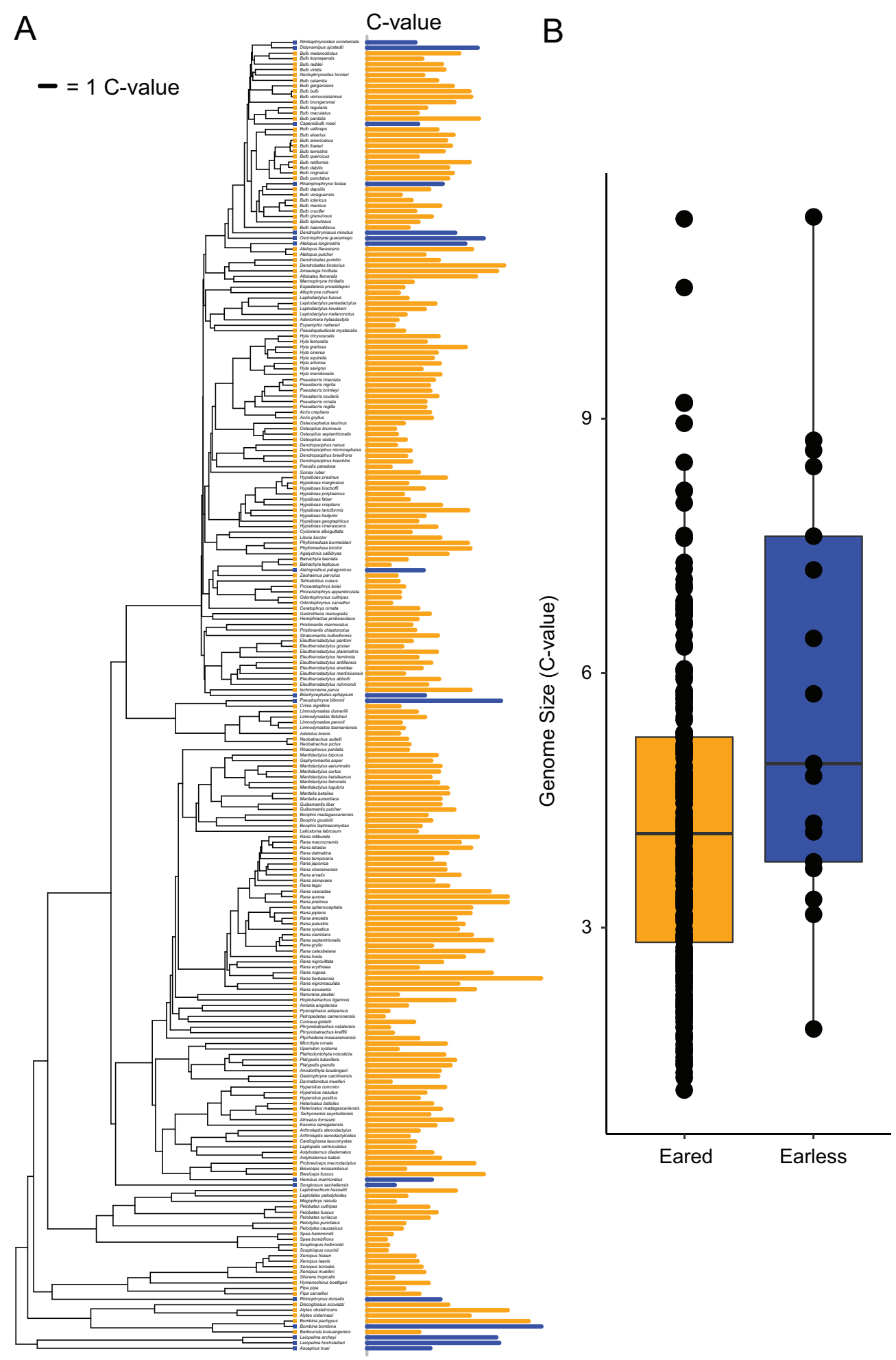

Figure 2: Earless species (blue) have larger average genomes than species that have a full middle ear bone (orange). A, Phylogeny (Pyron $2014 a, 2014 b$ ) showing the relationships between the eared and earless species included in this study and their estimated genome sizes (indicated by the bar length to the right of each species name). $B$, Boxplot with all data points overlaid showing the differences in genome size between eared and earless species. 


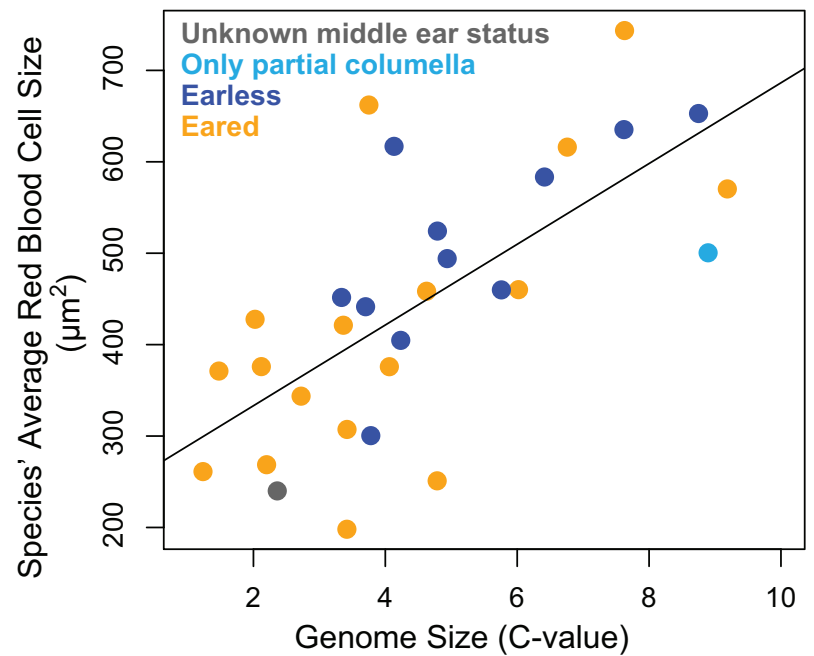

Figure 3: Relationship between genome size and red blood cell size. We found a positive relationship between genome size and red blood cell size among 30 anuran species that are eared (orange), are earless (blue), have partial columella present (light blue), or lack information about columella presence/absence (gray).

paedomorphosis and tympanic middle ear loss alone or in combination with postdisplacement, another form of paedomorphosis that would postpone tympanic middle ear development (relative to other traits), making it particularly vulnerable to paedomorphic loss. For example, the true toad family (Bufonidae) has the largest number of middle ear loss events and may be particularly susceptible to tympanic middle ear loss when development rate is slowed, since bufonids already have prolonged middle ear development in comparison to other anuran species (Sedra and Mi- chael 1959; Hetherington 1987; Womack et al. 2018b). Furthermore, although neoteny does not preclude truncated juvenile development (progenesis), comparative analyses of skulls did not find evidence that the columella is often lost with other late-forming traits (Womack et al. 2018a), as would be expected if progenesis were involved. More studies investigating the development of closely related eared and earless species across ear loss events will allow us to determine whether genome size and heterochrony are mediating earlessness via similar or disparate mechanisms among earless lineages.

\section{Support for Cell Size to Metamorph Body Size Ratio Affecting Tympanic Middle Ear Loss}

This study is the first phylogenetically informed test of whether small body sizes in combination with large cell sizes constrain structure development in anurans. We found that larger genome size (and associated larger cell sizes) are associated with middle ear loss, and we also find that earless species have smaller minimum body sizes at metamorphosis (when the middle ear begins to form). These findings are consistent with the hypothesis that larger cell sizes and smaller body size at metamorphosis could contribute to middle ear loss in anurans. Although the effects of larger cell size and small body size have mostly been discussed in relation to late-developing traits in salamanders (Hanken 1983; Wake 1991; Hanken and Wake 1993), we cannot rule out the possibility that large cell size and small body size similarly affect tympanic middle ear development in anurans. However, the maximum body sizes recorded for earless species at metamorphosis only trend smaller than the maximum recorded body
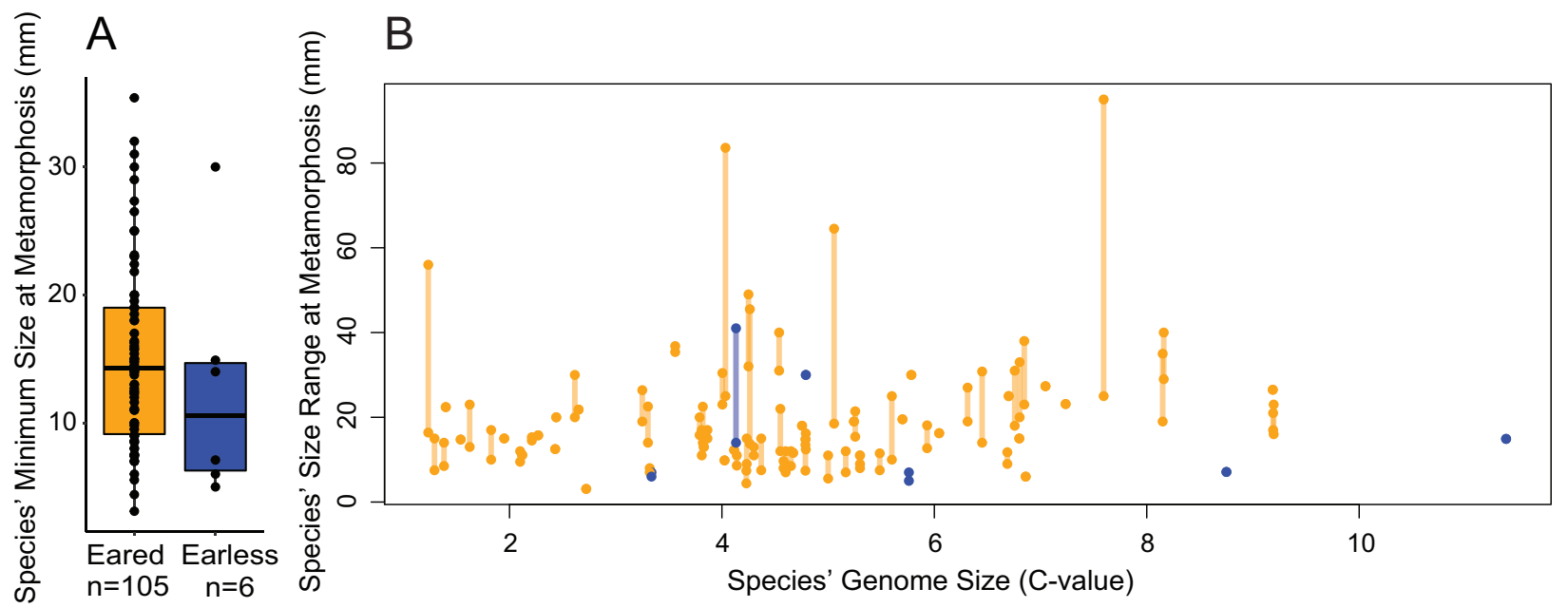

Figure 4: Earless species (blue) have smaller minimum and maximum body sizes at metamorphosis than species that have a full middle ear bone (orange). A, Boxplot showing that earless species have smaller minimum body size at metamorphosis than eared species. $B$, Scatterplots showing the relationship between genome size and size at metamorphosis for eared (orange) and earless (blue) species. 
size for eared metamorphs, introducing the possibility that earless species may not be constrained to metamorph body sizes that differ from eared species. Data on size at metamorphosis (when the middle ear starts to form) exist for only six earless species, limiting our ability to generalize our findings across the many additional species with tympanic middle ear loss. However, size at metamorphosis is positively correlated with adult body size among anuran species (Werner 1986), and earless anuran species tend to have small adult body sizes (Hetherington 1992; M. C. Womack, unpublished data), suggesting that small body size at metamorphosis in earless species may be a more widespread association. Our work constitutes the first phylogenetic comparison that supports a possible association among increased cell size, small body size, and loss of a late-forming amphibian structure.

\section{Other Potential Ecological and Developmental Contributors to Tympanic Middle Ear Loss}

Earless species lack shared environmental, life-history, or ecological traits, providing little evidence for alternative hypotheses to explain the repeated evolutionary loss of the anuran tympanic middle ear. In addition to a broad range in development rate, anurans show striking variation in development mode. Although most anurans undergo larval development where embryos hatch from an egg and then have a free-swimming tadpole stage, some species undergo direct development, in which development from embryo to froglet is completed with the egg, and other species are viviparous, such that eggs are retained inside the female until they develop into froglets. Although we analyzed only size at metamorphosis and development rate for earless larval species in this study, earless species with known genome sizes in our study include three direct-developing species and a single viviparous species (development mode data from Oliveira et al. 2017). Thus, tympanic middle ear loss occurs within species of all development modes. Furthermore, a recent study found no relationship between genome size and direct development (Liedtke et al. 2018), indicating that development mode is not a confounding variable in our analysis of the relationship between genome size and tympanic middle ear loss. Whether earless direct-developing species have smaller body sizes at hatching compared with eared direct-developing species remains to be tested.

We also lack obvious evidence for ecological or environmental selection pressures contributing to earlessness. The earless species within this study inhabit a variety of microhabitats (including aquatic, burrowing, terrestrial, and torrential; Moen and Wiens 2017) and are geographically widespread (AmphibiaWeb 2019). Additionally, although a few earless species lack voice boxes (e.g., Leiopelma archeyi and L. hochstetteri [Trewavas 1932]), many earless species call (Rhinophrynus dorsalis [Porter 1962], Hemisus marmoratus
[Passmore and Carruthers 1995], Nasikabatrachus sahyadrensis [Thomas et al. 2014], Nimbaphrynoides occidentalis [Sandberger et al. 2010], Osornophryne guacamayo [Gluesenkamp and Acosta 2001], Sooglossus sechellensis [Nussbaum et al. 1982]). Furthermore, calls of earless species often have dominant frequencies above $1 \mathrm{kHz}$ (e.g., Hemisus marmoratus, 3.5-4.2 kHz [Passmore and Carruthers 1995]; Nasikabatrachus sahyadrensis, $1.2-1.3 \mathrm{kHz}$ [Thomas et al. 2014]; Nimbaphrynoides occidentalis, $3.1 \mathrm{kHz}$ [mean frequency; Sandberger et al. 2010]; Osornophryne guacamayo, $3.2 \mathrm{kHz}$ [Gluesenkamp and Acosta 2001]; Sooglossus sechellensis, $2.7 \mathrm{kHz}$ [Nussbaum et al. 1982]), in the frequency range where earlessness is associated with hearing deficits (Womack et al. 2017). Thus, little evidence implicates shared selection pressures related to the ecology of earless species in the repeated loss of the anuran tympanic middle ear; however, these alternative hypotheses remain to be tested at a broader phylogenetic scale.

\section{Concluding Remarks}

This study highlights potential developmental and morphological consequences of genome size evolution in anurans. Here we estimated the genome size of 22 anuran species and provide the largest comparative study of genome size in relation to a trait loss. Our findings support the positive relationship among genome size, larval period length, and cell size within Anura while accounting for phylogenetic relationships. We also show that earless species tend to have larger genomes than their eared relatives, implicating slowed development and/or cell size constraints during metamorphosis in convergent tympanic middle ear loss in anurans.

\section{Acknowledgments}

We thank the people who helped obtain the wild-caught animals for this study, Elicio Tapia, Luis Coloma, and Amanda Delgado, with special thanks to Lola Guarderas and Amanda Delgado for facilitating collection and transportation permits. We thank the Smithsonian National Museum of Natural History $(\mathrm{NMNH})$ for allowing us access to specimens used in this study and Addison Wynn for help within the NMNH collections. We thank Stanley Sessions for guidance on Feulgen staining. We also thank two anonymous reviewers and an associate editor, Dan Rabosky, for their helpful comments. Funding for this study was provided by the US National Science Foundation (NSF IOS-13503461350346, PRFB-1611752).

Statement of authorship: M.C.W. contributed to the conception of the study, data collection, data analysis, and writing. M.J.M. contributed to data collection, data analysis, and writing. K.L.H. contributed to the conception of the study, data analysis, and writing. 


\section{Literature Cited}

Alberch, P., S. J. Gould, G. F. Oster, and D. B. Wake. 1979. Size and shape in ontogeny and phylogeny. Paleobiology 5:296-317.

Alvarez, D., and A. G. Nicieza. 2002. Effects of temperature and food quality on anuran larval growth and metamorphosis. Functional Ecology 16:640-648.

AmphibiaWeb. 2019. Accessed February 12, 2019. http://amphibiaweb .org. University of California, Berkeley.

Bachmann, K. 1972. Nuclear DNA and developmental rate in frogs. Quarterly Journal of the Florida Academy of Sciences 35:225-231.

Bennett, M. D. 1977. The time and duration of meiosis. Philosophical Transactions of the Royal Society B 277:201-277.

Buchholz, D. R., and T. B. Hayes. 2002. Evolutionary patterns of diversity in spadefoot toad metamorphosis (Anura: Pelobatidae). Copeia 2002:180-189.

Camper, J. D., L. A. Ruedas, J. W. Bickham, and J. R. Dixon. 1993. The relationship of genome size with developmental rates and reproductive strategies in five families of Neotropical bufonoid frogs. Life Science Advances 12:79-87.

Chipman, A. D., O. Khaner, A. Haas, and E. Tchernov. 2001. The evolution of genome size: what can be learned from anuran development? Journal of Experimental Zoology 291:365-374.

Collins, J. P. 1979. Intrapopulation variation in the body size at metamorphosis and timing of metamorphosis in the bullfrog, Rana catesbeiana. Ecology 60:738-749.

Gluesenkamp, A. G., and N. Acosta. 2001. Sexual dimorphism in Osornophryne guacamayo with notes on natural history and reproduction in the species. Journal of Herpetology 35:148-151.

Goin, O. B., C. J. Goin, and K. Bachmann. 1968. DNA and amphibian life history. Copeia 3:532-540.

Gould, S. J. 1977. Ontogeny and phylogeny. Harvard University Press, Cambridge, MA.

Gregory, T. R. 2000. Nucleotypic effects without nuclei: genome size and erythrocyte size in mammals. Genome 43:895-901.

. 2001. The bigger the C-value, the larger the cell: genome size and red blood cell size in vertebrates. Blood Cells, Molecules, and Diseases 27:830-843.

- 2002a. A bird's eye view of the C-value enigma: genome size, cell size, and metabolic rate in the class aves. Evolution 56:121-130.

2002b. Genome size and developmental complexity. Genetica 115:131-146.

_. 2018. Animal Genome Size Database. http://www.genome size.com.

Hanken, J. 1983. Miniaturization and its effects on cranial morphology in plethodontid salamanders, genus Thorius (Amphibia, Plethodontidae). II. The fate of the brain and sense organs and their role in skull morphogenesis and evolution. Journal of Morphology 177:255-268.

Hanken, J., and D. B. Wake. 1993. Miniaturization of body size: organismal consequences and evolutionary significance. Annual Review of Ecology and Systematics 24:501-519.

Hardie, D., T. Gregory, and P. Herbert. 2002. From pixels to picograms: a beginner's guide to genome quantification by Feulgen image analysis densitometry. Journal of Histochemistry and Cytochemistry 50:735-749.

Hardie, D., and P. Herbert. 2003. The nucleotypic effects of cellular DNA content in cartilaginous and ray-finned fishes. Genome 46:683-706.

Hetherington, T. E. 1987. Timing of development of the middle ear of Anura (Amphibia). Zoomorphology 106:289-300.
1992. The effects of body size on the evolution of the amphibian middle ear. Pages 421-437 in D. B. Webster, A. N. Popper, and R. R. Fay, eds. The evolutionary biology of hearing. Springer, New York. Horner, H. A., and H. C. Macgregor. 1983. C value and cell volume: their significance in the evolution and development of amphibians. Journal of Cell Science 63:135-146.

Horowitz, S. S., J. A. Chapman, U. Kaya, and A. M. Simmons. 2001. Metamorphic development of the bronchial columella of the larval bullfrog (Rana catesbeiana). Hearing Research 154:12-25.

Jaslow, A. P., T. E. Hetherington, and R. E. Lombard. 1988. Structure and function of the amphibian middle ear. Pages 69-91 in B. Fritzsch, M. J. Ryan, W. Wilczynski, T. E. Hetherington, and W. Walkowiak, eds. The evolution of the amphibian auditory system. J. Wiley, New York.

Kupferberg, S. J., J. C. Marks, and M. E. Power. 1994. Effects of variation in natural algal and detrital diets on larval anuran ( $H y l a$ regilla) life-history traits. Copeia 2:446-457.

Liedtke, H. C., D. J. Gower, M. Wilkinson, and I. Gomez-Mestre. 2018. Macroevolutionary shift in the size of amphibian genomes and the role of life history and climate. Nature Ecology and Evolution 2:1792.

Lombard, R. E., and I. R. Straughan. 1974. Functional aspects of anuran middle ear structures. Journal of Experimental Biology 61:71-93.

Manley, G. A. 2010. An evolutionary perspective on middle ears. Hearing Research 263:3-8.

Marian, M. P., and T. J. Pandian. 1985. Effect of temperature on development, growth and bioenergetics of the bullfrog tadpole Rana tigrina. Journal of Thermal Biology 10:157-161.

McNamara, K. J. 1986. A guide to the nomenclature of heterochrony. Journal of Paleontology 60:4-13.

Moen, D. S., and J. J. Wiens. 2017. Microhabitat and climatic niche change explain patterns of diversification among frog families. American Naturalist 190:29-44.

Moreira, G., and A. P. Lima. 1991. Seasonal patterns of juvenile recruitment and reproduction in four species of leaf litter frogs in central Amazonia. Herpetologica 47:295-300.

Mueller, R. L., T. R. Gregory, S. M. Gregory, A. Hsieh, and J. L. Boore. 2008. Genome size, cell size, and the evolution of enucleated erythrocytes in attenuate salamanders. Zoology 111:218-230.

Nussbaum, R. A., A. Jaslow, and J. Watson. 1982. Vocalization in frogs of the family Sooglossidae. Journal of Herpetology 16:198-203.

Oeldorf, E., M. Nishiokac, and K. Bachmann. 1978. Nuclear DNA amounts and developmental rate in holarctic anura. Journal of Zoological Systematics and Evolutionary Research 16:216-224.

Ohno, S. 1970. The enormous diversity in genome sizes of fish as a reflection of nature's extensive experiments with gene duplication. Transactions of the American Fisheries Society 99:120-130.

Oliveira, B. F., V. A. São-Pedro, G. Santos-Barrera, C. Penone, and G. C. Costa. 2017. AmphiBIO, a global database for amphibian ecological traits. Scientific Data 4:170123.

Olmo, E., and A. Morescalchi. 1975. Evolution of the genome and cell sizes in salamanders. Experientia 31:804-806.

. 1978. Genome and cell size in frogs: a comparison with salamanders. Experientia 34:44-46.

Passmore, N. I., and V. C. Carruthers. 1995. South African frogs. 2nd ed. Southern Book Publishers, Johannesburg.

Pereyra, M. O., M. C. Womack, J. S. Barrionuevo, B. L. Blotto, D. Baldo, M. Targino, J. J. Ospina-Sarria, et al. 2016. The complex evolutionary history of the tympanic middle ear in frogs and toads (Anura). Scientific Reports 6:34130. 
Pfennig, D. W., A. Mabry, and D. Orange. 1991. Environmental causes of correlations between age and size at metamorphosis in Scaphiopus multiplicatus. Ecology 72:2240-2248.

Pinheiro, J., D. Bates, S. DebRoy, D. Sarkar, and R Core Team. 2017. nlme: linear and nonlinear mixed effects models. $\mathrm{R}$ package version 3.1-131. https://CRAN.R-project.org/package $=$ nlme.

Porter, K. R. 1962. Mating calls and noteworthy collections of some Mexican amphibians. Herpetologica 18:165-171.

Pyron, R. A. 2014a. Biogeographic analysis reveals ancient continental vicariance and recent oceanic dispersal in amphibians. Systematic Biology 63:779-797.

. 2014b. Data from: Biogeographic analysis reveals ancient continental vicariance and recent oceanic dispersal in amphibians Systematic Biology, Dryad Digital Repository, https://doi.org/10.5061 /dryad.jm453.

R Core Team. 2017. R: a language and environment for statistical computing. R Foundation for Statistical Computing, Vienna. http://www .R-project.org/.

Revell, L. J. 2010. Phylogenetic signal and linear regression on species data. Methods in Ecology and Evolution 1:319-329.

. 2012. phytools: an R package for phylogenetic comparative biology (and other things). Methods in Ecology and Evolution 3:217-223. https://doi.org/10.1111/j.2041-210X.2011.00169.x.

Richter-Boix, A., M. Tejedo, and E. L. Rezende. 2011. Evolution and plasticity of anuran larval development in response to desiccation a comparative analysis. Ecology and Evolution 1:15-25.

Roth, G., J. Blanke, and D. B. Wake. 1994. Cell size predicts morphological complexity in the brains of frogs and salamanders. Proceedings of the National Academy of Sciences of the USA 91:4796-4800.

Roth, G., and W. Walkowiak. 2015. The influence of genome and cell size on brain morphology in amphibians. Cold Spring Harbor Perspectives in Biology 7:a019075.

Sandberger, L., A. Hillers, J. Doumbia, N. S. Loua, C. Brede, and M. O. Roedel. 2010. Rediscovery of the Liberian Nimba toad, Nimbaphrynoides liberiensis (Xavier, 1978) (Amphibia: Anura: Bufonidae), and reassessment of its taxonomic status. Zootaxa 2355:56-68.

Schindelin, J., I. Arganda-Carreras, E. Frise, V. Kaynig, M. Longair, T. Pietzsch, S. Preibisch, et al. 2012. Fiji: an open-source platform for biological-image analysis. Nature Methods 9:676-682.

Sedra, S. N., and M. I. Michael. 1959. The ontogenesis of the sound conducting apparatus of the Egyptian toad, Bufo regularis Reuss, with a review of this apparatus in Salientia. Journal of Morphology 104:359-375.

Sessions, S. K., and A. Larson. 1987. Developmental correlates of genome size in plethodontid salamanders and their implications for genome evolution. Evolution 41:1239-1251.

Shuter, B. J., J. E. Thomas, W. D. Taylor, and A. M. Zimmerman. 1983. Phenotypic correlates of genomic DNA content in unicellular eukaryotes and other cells. American Naturalist 122:26-44.

Smirnov, S. V. 1991. The anuran middle ear: developmental heterochronies and adult morphology diversification. Belgian Journal of Zoology 121:99-110.

Szarski, H. 1970. Changes in the amount of DNA in cell nuclei during vertebrate evolution. Nature 226:651.
1983. Cell size and the concept of wasteful and frugal evolutionary strategies. Journal of Theoretical Biology 105:201-209.

Thomas, A., R. Suyesh, S. D. Biju, and M. A. Bee. 2014. Vocal behavior of the elusive purple frog of India (Nasikabatrachus sahyadrensis), a fossorial species endemic to the Western Ghats. PLoS ONE 9:e84809.

Trewavas, E. 1932. The hyoid and larynx of the Anura. Philosophical Transactions of the Royal Society B 222:401-527.

van Eeden, J. A. 1951. The development of the chondrocranium of Ascaphus truei Stejneger with special reference to the relations of the palatoquadrate to the neurocranium. Acta Zoologica 32:41-176.

Vinogradov, A. E. 1995. Nucleotypic effect in homeotherms: bodymass-corrected basal metabolic rates of mammals is related to genome size. Evolution 49:1249-1259.

1997. Nucleotypic effect in homeotherms: body-mass independent resting metabolic rate of passerine birds is related to genome size. Evolution 51:220-225.

1999. Genome in toto. Genome 42:361-362.

Vorobyeva, E., and S. Smirnov. 1987. Characteristic features in the formation of anuran sound-conducting systems. Journal of Morphology 192:1-11.

Wake, D. B. 1966. Comparative osteology and evolution of the lungless salamanders, family Plethodontidae. Memoirs of the Southern California Academy of Sciences 4:1-111.

- 1991. Homoplasy: the result of natural selection, or evidence of design limitations? American Naturalist 138:543-567.

- 2009. What salamanders have taught us about evolution. Annual Review of Ecology, Evolution, and Systematics 40:333-352.

Walker, L., A. Spotorno, and J. Sans. 1991. Genome size variation and its phenotypic consequences in Phyllotis rodents. Hereditas 115:99-107.

Werner, E. E. 1986. Amphibian metamorphosis: growth rate, predation risk, and the optimal size at transformation. American Naturalist 128:319-341.

White, M. M., and I. A. McLaren. 2000. Copepod development rates in relation to genome size and $18 \mathrm{~S}$ rDNA copy number. Genome 43:750-755.

Womack, M. C., J. Christensen-Dalsgaard, L. A. Coloma, J. C. Chaparro, and K. L. Hoke. 2017. Earless toads sense low frequencies but miss the high notes. Proceedings of the Royal Society B 284:20171670.

Womack, M. C., T. S. Fiero, and K. L. Hoke. 2018a. Trait independence primes convergent trait loss. Evolution 72:679-687.

Womack, M. C., M. J. Metz, and K. L. Hoke. 2019. Larger genomes linked to slower development and loss of late-developing traits. American Naturalist, Dryad Digital Repository, https://doi.org/10 $.5061 /$ dryad.k02pq01.

Womack, M. C., J. L. Stynoski, M. K. Voyles, L. A. Coloma, and K. L. Hoke. 2018b. Prolonged middle ear development in Rhinella horribilis. Journal of Morphology 279:1518-1523.

Associate Editor: Daniel L. Rabosky Editor: Russell Bonduriansky 